\title{
Reflets
}

Revue d'intervention sociale et communautaire

\section{Une expérience de stage novatrice en nutrition : facteurs facilitants et défis}

\section{Lynn Casimiro, Geneviève Grenier, Manon Tremblay, Mélanie Desmarais et Denise Beaulieu}

Volume 20, numéro 1, printemps 2014

La formation pratique : allier milieux de pratique et milieux

d'enseignement

URI : https://id.erudit.org/iderudit/1025798ar

DOI : https://doi.org/10.7202/1025798ar

Aller au sommaire du numéro

Éditeur(s)

Reflets, Revue d'intervention sociale et communautaire

ISSN

1203-4576 (imprimé)

1712-8498 (numérique)

Découvrir la revue

Citer cet article

Casimiro, L., Grenier, G., Tremblay, M., Desmarais, M. \& Beaulieu, D. (2014). Une expérience de stage novatrice en nutrition : facteurs facilitants et défis. Reflets, 20(1), 130-140. https://doi.org/10.7202/1025798ar 


\section{Une expérience de stage novatrice en nutrition : facteurs facilitants et défis}

Lynn Casimiro, Ph.D.

Directrice de l'enseignement professionnel et interprofessionnel, Hôpital Montfort, Ottawa.

Geneviève Grenier, Dt.P/RD, MSc.

Coordonnatrice de la formation pratique, Programme de baccalauréat spécialisé en sciences de la nutrition, Université d'Ottawa

Manon Tremblay, Ph.D.

Directrice du Centre de développement professionnel continu Prométhée.

Hôpital Montfort, Ottawa

Mélanie Desmarais, MPA (c).

Chargée de projets en développement pédagogique, Hôpital Montfort, Ottawa

Denise Beaulieu, Ph.D.

Consultation M.D. Beaulieu, Gatineau

\section{Introduction}

Créé en 2007, le Programme en sciences de la nutrition de l'Université d'Ottawa a pour objectif de "former, en français, des étudiants bilingues qui rempliront les exigences requises à l'examen d'entrée à la profession de diététiste, dans un environnement stimulant et de favoriser la transmission des connaissances de pointe en sciences afin de combler le besoin actuel en professionnels de la santé capables d'offrir des services en français et en anglais » (Université d'Ottawa, 2007). Depuis sa 
création, le programme est inévitablement confronté à la pénurie de professionnels de la santé qualifiés, capables de participer à la mise en œuvre d'un programme de formation en français. En effet, le Programme de baccalauréat spécialisé en sciences de la nutrition de l'Université d'Ottawa exige que les étudiantes et les étudiants complètent des stages supervisés en milieux cliniques et doit ainsi avoir recours à des professionnels compétents afin d'agir à titre de superviseurs, notamment dans les communautés francophones en situation minoritaire (CNFS, 2006).

Les stages font partie intégrante de tout programme de formation des professionnels de la santé et des services sociaux. Essentielle au processus de professionnalisation des divers intervenants, la formation pratique permet de développer différentes compétences, d'établir le lien entre la théorie et la pratique professionnelle, d'apprendre à travailler en collaboration avec les membres d'une équipe, bref, de se forger une identité professionnelle. Continuer à former la relève exige innovation et efficacité.

Un second défi s'ajoute au simple recrutement des stages pratiques en nutrition, celui de trouver des stages en milieux francophones. Comme le mentionne le Cadre de référence pour la formation à l'offre active des services de santé en français produit par le Consortium national en santé (Lortie, Lalonde et Bouchard, 2012), le caractère social de la mission des universités contribue à la vitalité des communautés francophones. Des approches permettant de superviser un plus grand nombre de stagiaires à la fois s'imposent alors dans ce contexte de pénurie. Ainsi, les étudiantes et les étudiants francophones et francophiles peuvent parler, apprendre et ultérieurement travailler en français. Par conséquent, leur offrir des stages en français s'avère une intervention cruciale dans le processus identitaire de professionnalisation; en effet, ces stages forment au langage professionnel français et encouragent les étudiantes et les étudiants inscrits au programme de nutrition à offrir activement des services dans cette langue.

Face à ces défis, le Programme de baccalauréat spécialisé en sciences de la nutrition de l'Université d'Ottawa et l'Hôpital Montfort, un site affilié de formation clinique, a développé une 
nouvelle approche afin d'augmenter la capacité d'accueil de stagiaires. Cette approche combine des activités de supervision individuelle et de groupe et représente une première dans le cadre de stages en nutrition clinique offerts en milieu hospitalier.

Cet article a pour but de présenter le projet pilote comprenant deux stages en nutrition qui ont eu lieu à l'hiver et au printemps de 2012 et plus particulièrement les résultats qui ont été obtenus à la suite de l'évaluation de cette nouvelle approche de formation. Cette expérience de stage a permis de dresser des lignes directrices qui pourront guider non seulement les éducatrices et les éducateurs en nutrition, mais également ceux et celles d'autres disciplines qui souhaitent mettre en œuvre des stages novateurs dans un milieu où il manque de professionnels pour assumer le rôle de superviseur de stage.

\section{Contexte de l'évaluation : problématique}

Afin de répondre aux exigences nationales pour l'entrée à la profession de diététiste, tous les étudiantes et étudiants inscrits au Programme de baccalauréat spécialisé en sciences de la nutrition de l'Université d'Ottawa - programme d'une durée de quatre ans - doivent compléter quatre stages de formation pratique : deux stages de douze semaines, l'un en gestion des services alimentaires et l'autre en nutrition clinique, ainsi que deux stages de six semaines en nutrition communautaire et en milieux variés.

En janvier 2012, le programme s'est retrouvé en pénurie de stages en nutrition clinique.Afin de contrer ce problème, l'Hôpital Montfort a accepté d'accueillir les sept personnes affectées par cette situation en plus des quatre stagiaires qui leur avaient déjà été confiés. Deux groupes d'étudiantes et étudiants ont été formés, l'un de cinq stagiaires et l'autre de six.

Étant donné que cette expérience était nouvelle pour le domaine de la nutrition et peu documentée, il était pertinent d'évaluer le processus et l'implantation de ce projet, de manière à tirer des leçons et à dresser des lignes directrices qui permettraient de reproduire ce type de stage (Monette, Charrette et Jobin, 
1995). L'évaluation formative touchait au déroulement du stage, à savoir à son climat et aux apprentissages des participantes et des participants. Les données de cette évaluation ont servi à vérifier s'il $\mathrm{y}$ avait adéquation entre les activités et les objectifs d'apprentissage et si les activités rejoignaient bien la clientèle visée. L'évaluation a cerné les facteurs de succès ainsi que les forces et les limites de la stratégie de supervision utilisée dans le cadre des stages.

\section{Le cadre opératoire des stages de nutrition clinique}

Chaque cohorte était sous la supervision d'une préceptricecoordonnatrice. Son rôle était de coordonner l'intégration et la rotation des stagiaires dans les différents secteurs ainsi que d'appuyer les autres préceptrices dans la supervision des stagiaires. Elle devait planifier et mener des rencontres individuelles avec les stagiaires afin d'assurer le suivi et la continuité dans leur cheminement personnel, en plus d'animer des rencontres de groupe. Elle assurait la liaison avec les instances pertinentes de l'établissement d'enseignement en vue de coordonner les activités et les évaluations périodiques. Chaque stagiaire était également sous la responsabilité de la diététiste couvrant la spécialité associée à chaque rotation, assurant ainsi sa supervision au quotidien et la transmission de connaissances dans le cadre particulier des problématiques de santé.

La structure des stages, c'est-à-dire la rotation des stagiaires d'un service à l'autre, s'avérait complexe à organiser et à coordonner. Les services ciblés durant le projet ont été les services de médecine, de chirurgie, de cardiologie, de soins intensifs, de réadaptation, de santé mentale ainsi que la clinique externe. Ces services accueillaient tout au plus deux stagiaires à la fois selon leur capacité.

\section{Description des participants}

La première cohorte de stagiaires qui a réalisé son stage de janvier à avril était composée de quatre étudiantes et d'un étudiant. La seconde cohorte était composée de quatre étudiantes et de deux 
étudiants. Leur âge variait de 20 à 30 ans au moment du stage.Vu leur petit nombre, aucun autre détail n'est donné sur eux afin de respecter leur anonymat.

Les principaux critères de sélection de la préceptrice-coordonnatrice comprenaient une bonne connaissance du milieu et une solide identité professionnelle relativement à sa pratique. Un nombre minimal de cinq années d'expérience était requis. En effet, selon Ndoreraho et Martineau (2006), la durée de la phase de l'insertion professionnelle de cinq ans semble être une moyenne logique à la vue des différentes estimations faites à partir des diverses études consultées.

\section{Évaluation du projet : stratégies de collecte et d'analyse des données}

Le processus d'évaluation a été mené par une consultante en collaboration avec des représentants de l'Université d'Ottawa et de l'Hôpital Montfort. Les discussions de groupe de stagiaires et les entretiens semi-directifs ont été utilisés pour la collecte de données primaires. Chaque discussion a duré 90 minutes et était divisée en cinq parties distinctes : l'introduction; la description de l'expérience de stage; l'analyse des réponses; le repérage des facteurs d'influence; la conclusion. Les stagiaires ont inscrit sur des bouts de papier adhésifs de type post-it qu'ils collaient au mur les points forts de leur expérience de supervision en groupe ainsi que les points à améliorer. Puis, ils ont été invités à consulter et à regrouper par thèmes tous les bouts de papier adhésifs placés sur le mur. Une discussion s'en est suivie dans le but de cerner et de bien comprendre les commentaires et l'expérience des stagiaires. Un résumé des résultats des entretiens a été acheminé à toutes les personnes des groupes de discussion pour qu'elles puissent les valider.

Deux entretiens semi-dirigés auprès de la préceptrice-coordonnatrice des stages de nutrition clinique ont été menés à la fin de la période de stage de chaque cohorte afin de capter son 
appréciation globale de l'expérience de stage. Un autre entretien a été mené avec un responsable du programme de formation de la maison d'enseignement afin cerner sa perception.

La collecte de données secondaires a comporté deux pôles. Dans un premier temps, le procès-verbal de la rencontre des coordonnatrices de la formation pratique du Programme de baccalauréat spécialisé en sciences de la nutrition de l'Université d'Ottawa et des diététistes participantes a été analysé par la consultante qui n'avait pas participé à cette rencontre. Dans un second temps, les réponses aux différents formulaires d'évaluation ont été compilées et intégrées aux données analysées dans le cadre de l'évaluation.

\section{Tableau 1 : Types et nombre de formulaires remplis et compilés dans le cadre de l'étude}

\begin{tabular}{|l|c|c|}
\hline $\begin{array}{l}\text { Formulaires remplis par les stagiaires sur différentes } \\
\text { dimensions de leur expérience de stage en nutrition } \\
\text { clinique }\end{array}$ & $\begin{array}{l}\text { Nombre de formulaires } \\
\text { compilés }\end{array}$ \\
\hline & $\mathbf{1}^{\text {re }}$ cohorte & $\mathbf{2}^{\mathbf{e}}$ cohorte \\
\hline $\begin{array}{l}\text { Formulaire d'évaluation des stages de l'Hôpital Montfort, } \\
\text { administré par la Direction des affaires universitaires. }\end{array}$ & 3 & 5 \\
\hline $\begin{array}{l}\text { Formulaire d'évaluation des stages du Programme de baccalauréat } \\
\text { spécialisé en sciences de la nutrition de l'Université d'Ottawa visant } \\
\text { à évaluer la satisfaction du stagiaire. }\end{array}$ & 4 & 1 \\
\hline $\begin{array}{l}\text { Sondage sur l'atteinte des compétences de collaboration } \\
\text { interprofessionnelles à l'Hôpital Montfort. }\end{array}$ & 5 & 6 \\
\hline
\end{tabular}

\section{Analyse des données}

L'analyse des données recueillies a permis de regrouper les résultats sous deux grands thèmes : l'organisation générale des stages et les activités pédagogiques. L’organisation générale comprend la coordination, l'établissement des horaires de rotation et les autres aspects logistiques tels les locaux mis à la disposition des stagiaires. 


\section{Organisation générale du stage}

L'intégration de cinq ou six stagiaires au sein d'une organisation aussi complexe qu'un milieu hospitalier soulève de nombreux défis. Voici un aperçu des éléments dont la préceptrice-coordonnatrice devait tenir compte afin de coordonner efficacement les rotations des stagiaires et les autres activités pédagogiques du stage :

- les horaires de travail et de vacances des autres diététistes et le faible taux de patients hospitalisés durant les mois de juin et de juillet pour le stage de la deuxième cohorte;

- les caractéristiques particulières de chaque service. L'équipe d'encadrement juge préférable de donner aux stagiaires plus de temps pour s'intégrer à l'hôpital avant d'entreprendre une rotation complexe tels les soins intensifs;

- la recherche d'un équilibre satisfaisant entre les activités cliniques et celles visant l'établissement de liens entre la théorie et la pratique;

- les exigences du programme de nutrition qui demande que les étudiantes et les étudiants soient exposés à certaines spécialités pour répondre aux exigences d'entrée à la profession de diététiste (par exemple, la nutrition entérale).

\section{Activités pédagogiques}

Les résultats de l'évaluation démontrent que les deux cohortes de stagiaires ont tiré profit d'une combinaison d'activités pédagogiques propices à l'atteinte de leurs objectifs d'apprentissage. Ils démontrent également que des améliorations ont été apportées à la structure et au contenu des activités offertes à la deuxième cohorte de stagiaires, témoignant ainsi de la volonté de l'équipe d'encadrement des stages à apporter les changements qui s'imposaient à la suite du stage de la première cohorte.

\section{La semaine de formation interprofessionnelle}

Tous les stagiaires ont fortement apprécié la première semaine de stage en formation interprofessionnelle. Amorcer ainsi le stage a facilité l'intégration des stagiaires au milieu. Le contenu des 
activités et l'encadrement offert par l'équipe de formation ont été jugés appropriés et de grande qualité par les deux cohortes.

\section{Le modèle de supervision adopté}

Le modèle de supervision combinant des activités de groupe et des activités individuelles a été considéré comme très efficace par les stagiaires. D'une part, la présence du groupe a été une source de soutien et un lieu propice à de nouveaux apprentissages. D'autre part, la supervision individuelle leur permettait de se centrer sur leurs besoins personnels d'apprentissage et de traiter de questions plus délicates. Dans le formulaire d'évaluation des stages, une étudiante indique comme activité la plus satisfaisante l'« encadrement-supervision idéal pour arriver à compléter un stage aussi exigeant ». Sous-tendus par un modèle d'apprentissage d'approche par problème, les stagiaires travaillaient ensemble sur un cas qu'ils devaient étudier en profondeur. Le même cas pouvait se complexifier au fil du temps et être étudié pendant plusieurs semaines.

\section{Qualité et style de supervision individuelle}

Les styles de supervision individuelle offerts par les différentes préceptrices ont été très appréciés des stagiaires qui ont souligné leur compétence, leur professionnalisme et leur engagement. Dans l'ensemble, leur approche de supervision semble avoir favorisé le développement des capacités de réflexion critique et de jugement. La consultation des feuilles de commentaires des formulaires d'évaluation des compétences atteste du ton positif, mais sans complaisance des préceptrices qui mettent l'accent sur les forces tout en soulignant avec tact les points à améliorer. Cela concorde avec les propos des stagiaires qui ont exprimé leur appréciation du ton et du contenu des commentaires des préceptrices.

\section{Processus d'évaluation}

Les stagiaires des deux cohortes ont souligné la difficulté de composer avec les attentes diverses et nombreuses de la part des différentes préceptrices. De plus, il est possible que toutes 
les activités de supervision aient créé une sorte d'effet de «surencadrement » qui pourrait se résorber en clarifiant davantage le rôle de chacun.

Les outils et les grilles d'autoévaluation et d'évaluation par les pairs utilisés dans le cadre des activités de supervision ont fait l'objet de certaines suggestions. Tous ont mentionné l'utilité de réfléchir sur leurs points forts et leurs points faibles, mais certains, dont plusieurs de la deuxième cohorte, ont indiqué qu'ils devaient faire cette analyse trop fréquemment et le faire une ou deux fois lors de chaque rotation pouvait suffire selon eux.

\section{Appréciation globale du stage}

La majorité des stagiaires dénotent une appréciation générale favorable et une compréhension des contraintes à laquelle les équipes de l'hôpital et de l'établissement d'enseignement ont dû faire face. Les rapports et les formulaires d'évaluation indiquent en effet que leur expérience globale a été positive et qu'ils ont atteint leurs objectifs d'apprentissage.

\section{Discussion : le modèle de supervision et ses facteurs de succès}

Le modèle de supervision employé était un modèle " mixte ", car il comportait des activités de supervision individuelle et de groupe. Un tel modèle a permis d'atteindre les objectifs pédagogiques de la formation en nutrition clinique tout en respectant l'organisation du travail et la capacité du milieu de stage. Ce modèle semble être un bon choix afin de combler l'insuffisance de lieux de stage, qui est particulièrement importante chez les francophones en situation minoritaire.

\section{Facteurs de succès}

Les facteurs qui ont contribué au succès de ce modèle de supervision dans le cadre de ces deux stages en nutrition clinique sont nombreux. La présence de la préceptrice-coordonnatrice a 
facilité l'intégration des apprentissages, les communications entre les stagiaires et les préceptrices et a permis à ces dernières de consacrer davantage de temps au développement des compétences professionnelles des stagiaires plutôt qu'à la coordination de l'ensemble des activités d'apprentissage. Ce modèle évite le dédoublement de tâches. La diversité des rotations et des styles de supervision a enrichi l'expérience vécue par les stagiaires. La préceptrice-coordonnatrice de stage qui a supervisé individuellement chaque stagiaire a permis d'assurer la continuité des apprentissages au fil des rotations et de faciliter l'établissement de liens entre la théorie et la pratique.

\section{Un aspect qui demande réflexion : le nombre de stagiaires}

Il ressort de l'analyse des données que le nombre de stagiaires en nutrition clinique pourrait difficilement dépasser six en raison de la taille du milieu hospitalier, du nombre de diététistes, de la structure organisationnelle de leur travail, qui est spécialisé et se concentre dans un service en particulier, ainsi qu'en raison de la taille optimale pour un groupe de discussion.

\section{Recommandations}

- conserver la semaine de formation interprofessionnelle;

- conserver le modèle mixte de supervision;

- approfondir la réflexion sur les activités de supervision de groupe;

- reconnaître la valeur ajoutée du rôle de préceptricecoordonnatrice;

- introduire une séance de supervision de groupe sur les relations avec les patients et les familles;

- poursuivre la documentation de cette nouvelle approche mixte de supervision de stage.

À cet effet, un guide répertoriant divers outils et conseils pratiques utilisés dans le cadre du stage en nutrition peut être consulté dans le site Web de l'Hôpital Montfort à l'adresse suivante : http://www.hopitalmontfort.com/sites/default/files/ PDF/guide_dactivite_pour_les_stages_de_groupe-nutrition.pdf 


\section{Conclusion}

L'évaluation de cette expérience de supervision de groupe dans le domaine de la nutrition clinique démontre que ce type de supervision dépasse la simple réponse à la pénurie de places de stages et de personnel qualifié apte à superviser. Lorsque mis en œuvre en tenant compte des limites qui lui sont inhérentes, ce modèle peut devenir une véritable source d'innovation pédagogique et une stratégie pour combler la pénurie de professionnels de la santé et des services sociaux dans les milieux minoritaires.

\section{Bibliographiques}

CONSORTIUM NATIONAL DE FORMATION EN SANTÉ (2006). Étude sur les besoins en formation clinique et en formation continue des professionnels de la santé des communautés francophones en situation minoritaire. Ottawa : CNFS Accès : http://cnfs.net/fr/publications-formation.php?rowstart $=10$

LORTIE, Lise, André J. LALONDE et Pier BOUCHARD (2012). Cadre de référence pour la formation à l'offre active des services de santé en français, Ottawa, Santé Canada.

MONETTE, Marcel, Mario CHARETTE et Ivan JOBIN (1995). Planifier et évaluer son intervention : module 3 : l'élaboration du programme, Cap-Rouge, Presses interuniversitaires.

NDORERAHO, Jean-Paul, et Stéphane MARTINEAU (2006). Recensions de thèses et de mémoires sur l'insertion professionnelle en enseignement, Trois-Rivières, QC : UQTR, LADIPE, 234 pages. Disponible en ligne : http://www.insertion.qc.ca/spip.php?article96.

UNIVERSITÉ D'OTTAWA (2007). Programme en sciences de la nutrition - Mission et vision, réf. du 5 mars 2014, Faculté des sciences de la santé, http://www.sante.uottawa.ca/nutrition/mission.htm

\section{Remerciement}

Ce projet (mise en œuvre et évaluation) a été rendu possible grâce au soutien du Consortium national de formation en santé (CNFS) et de la Faculté des sciences de la santé de l'Université d'Ottawa ainsi que de l'appui financier de Santé Canada. 